Originalveröffentlichung in: Baader, Hannah (Hrsg.): Im Agon der Künste : paragonales Denken, ästhetische Praxis und die Diversität der Sinne, München 2007, S. 542-562

\author{
VALESKA VON ROSEN
}

\title{
"Lieber Maler-Fürst als Foto-Graf«. Lois Renners fotografische Inszenierungen der Gattungen im Atelier*
}

\section{Paragone im 21. Jahrhundert?}

Sieht man vom Aspekt des Wettbewerbs ab und konzentriert sich stattdessen auf die Voraussetzungen des paragonalen Vergleichs der Gattungen in der Frühen Neuzeit, macht die Einbeziehung der zeitgenössischen Kunst in diesen Band durchaus Sinn. Denn diese Voraussetzungen bestehen im Ausloten der Möglichkeiten und Grenzen der spezifischen Visualität und Leistungsfähigkeit der verschiedenen Künste und sind damit Folge eines neuen medialen Bewußtseins in der Frühen Neuzeit, welches der Paragone gleichzeitig auch generierte. Gerade für die verhältnismäßig junge, erst seit etwa einer Generation als "hohe« Kunstform anerkannte und seither boomende Gattung der Fotografie ist ein entsprechendes Reflexionsbedürfnis und -niveau zu postulieren, was mit der Komplexität und Ambivalenz ihrer konstitutionellen Bedingungen zu erklären ist. ${ }^{1}$ Neben der "Universalität" dieser Kunstform, die ihr u. a. die Möglichkeit verleiht, alle anderen Gattungen abbilden und damit auch thematisieren zu können, ${ }^{2}$ ist hier der der Fotografie eigene paradoxale Charakter zu nennen. Er wird generiert durch die aus der "automatischen Genese ${ }^{3}$ resultierende topische Wirklichkeitsbezogenheit, Objektivität und "Wahrheit« der Fotografie einerseits, und ihrer durch Motivwahl, Ausschnittsetzung und Beleuchtung durch den Fotografen oder durch dessen Inszenierung der Welt vor der Linse bedingten Subjektivität andererseits. ${ }^{4}$ Dieser gattungsimmanente Dualismus ist der Fotografie seit ihrer Entstehung eingeschrieben, potenziert sich aber in den letzten Jahren durch die Möglichkeit der digitalen Bearbeitung und Erzeugung von Bildern.

* Für die Beantwortung zahlreicher Fragen danke ich Maribel Königer, Rupert Pfab und Lois Renner; Stefan Gronert und Philipp von Rosen für die kritische Lektüre des Manuskripts und wichtige Anregungen. Das Manuskript wurde im Sommer 2002 verfaßt und 2003, nach Erscheinen des Werkverzeichnisses von Renner überarbeitet. Danach erschienene Literatur konnte nicht mehr eingearbeitet werden.

1 Vgl. etwa Gronert (1999), S. 15.

2 Ein Argument, das im Paragonediskurs der Frühen Neuzeit von den Parteigängern der Malerei gegen die Skulptur in Anschlag gebracht wurde.

3 Bazin (1975), S. 24.

4 Vgl. für diesen Kontext das mit "Die Wahrheit in der Fotografie « überschriebene Kapitel von Groys (1997), S. 127-144 sowie die jeweiligen Einführungen von Wolfgang Kemp bzw. Hubertus von Amelunxen in: Kemp, von Amelunxen (1980-2000). 
Wenn ich die Frage nach der Reflexion dieser ontologischen Bedingungen der Fotografie hier mit Bezug auf das Euvre eines einzelnen Künstlers behandle, hat dies den Grund, daß sich die Arbeiten von Lois Renner ${ }^{5}$ hierfür geradezu paradigmatisch eignen. Denn Renner betreibt das Ausloten der Konstitutionsbedingungen der Fotografie, indem er den Paragone der Kunstgattungen in spielerischem Modus auf der Sujet-Ebene inszeniert und dies wiederum mit einem bestimmten künstlerischen Habitus verbindet. Er stilisiert sich nämlich selbst zum Maler, und zwar sowohl innerbildlich, wenn er sich in zahlreichen Werken als Maler "porträtiert", als auch in verbaler Form - ich verweise nur auf das Zitat im Obertitel. ${ }^{6}$ Diese Stilisierung manifestiert sich auch in dem besonderen Sujet seiner Arbeiten, das mit dem Atelier des Künstlers ein etabliertes Thema der okzidentalen Bildtradition aufgreift.

\section{Das Paragone-Tableau im Barberinischen Faun}

Keine zweite Arbeit Renners inszeniert die Gattungen im Atelier in derart komplexer Weise wie der Barberinische Faun 3000 aus dem Jahre 2000. Er hat die Maße $286 \times 350 \mathrm{~cm}$ und befindet sich im "museum kunst palast « in Düsseldorf (Abb. 1). ${ }^{7}$ Durch die Relationierung der drei Größen Künstleratelier, Künstler und

5 Renner wurde 1961 in Salzburg geboren, wo er auch eine Ausbildung absolvierte und das Studium aufnahm, das er an der Akademie in Düsseldorf fortsetze. Seit 1993 lebt er in Wien und Karlsruhe, wo er seit 2002 eine Professur für Fotografie inne hat. Neben einer ganzen Reihe eher essayistischer Ausstellungsbesprechen gibt es zu Renners Arbeiten folgende eingehendere Analysen: Trummer (1998), S. 131-143; Puvogel (1998), S. 144160 (der Inhalt dieses Beitrags ist in den Renner betreffenden Passagen im wesentlichen identisch mit ihrem 1994 in der Zeitschrift "Artis" erschienenen Aufsatz: "Das Atelier als Thema - Das Atelier als Bild und Raum); Metzger (2001), S. 20-31; Zeising (2002), S. 22-33, bes. 23-26 sowie nun das anläßlich der Ausstellung in der Landesgalerie am Oberösterreichischen Landesmuseum entstandene Werkverzeichnis des Künstlers: Lois Renner (2003); es enthält farbige Abbildungen aller Werke Renners, auf die ich jeweils verweise.

6 Es stammt aus einem Interview, das Maren Lübbke mit Renner geführt hat (Lübbke 1998, S. 17-32, hier 21) und überbeschrieb es bereits. Weil es für mein Thema des Paragone so geeignet ist, habe ich mir erlaubt, auch meinen Beitrag damit zu betiteln. Vgl. auch Renners Bemerkung im Interview mit Achim Hochdörfer, in: La casa, il corpo, il cuore (1999), S. 159-163, hier 160: "Ich sehe mich als Maler, der am Modell Bilder macht und die werden anschließend fotografiert".

7 Farbabbildung in: Lois Renner (2003), S. 261. Es handelt sich um zwei zusammenmontierte Color Prints, die im Diasec-Verfahren mit Plexiglas verklebt sind. Für alle technischen Informationen siehe Maribel Königer, Kommentiertes Verzeichnis der Bilder 1991-2002, in: Lois Renner (2003), S. 27-29. Die "3000« im Titel indiziert das Format; es gibt kleinere Varianten des Bildes, die entsprechend den Zusatz "500 « im Titel tragen; vgl. zu diesem Bild Zeising (2002); Geschichte der österreichischen Kunst (2001), S. 255; Ways of Worldmaking (2001), S. 122f. 


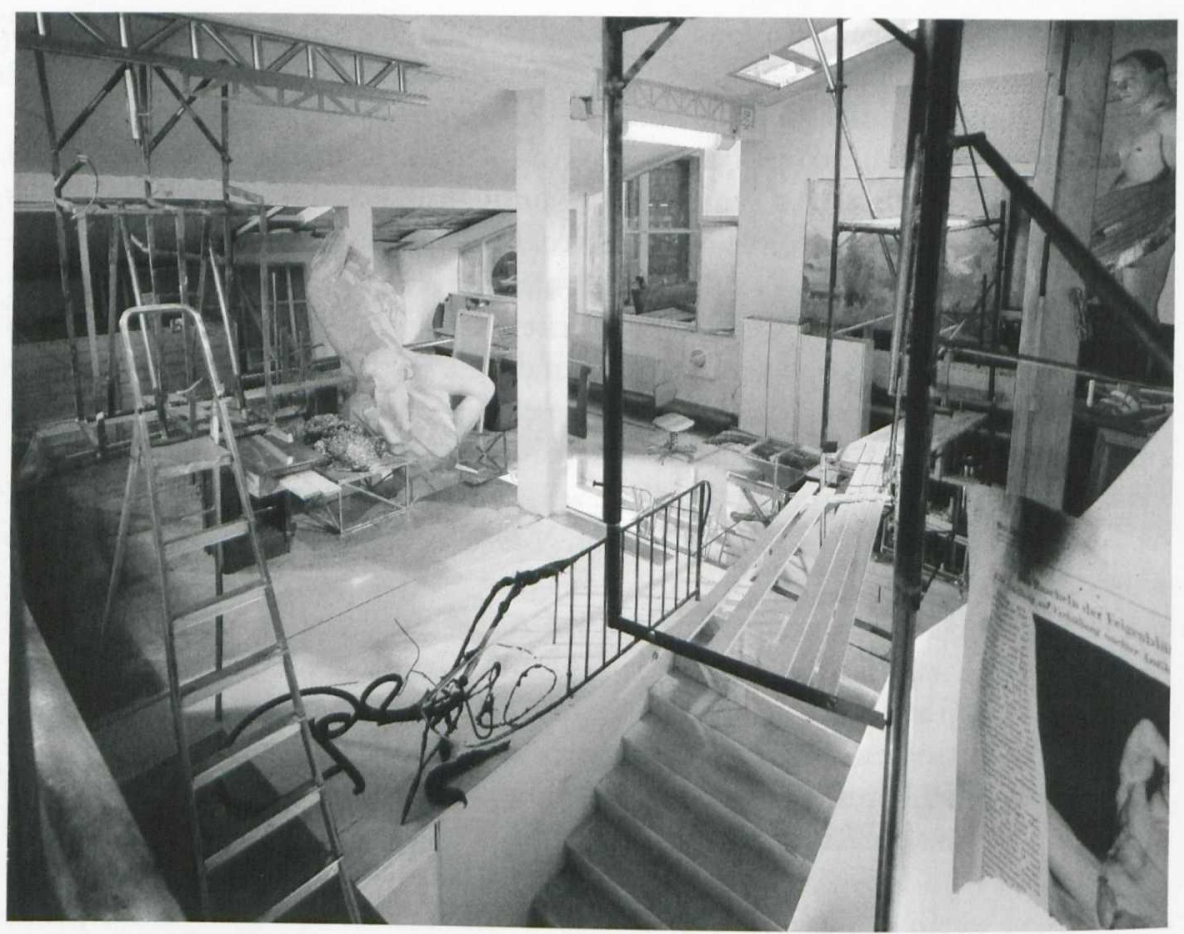

1. Lois Renner, Barberinischer Faun 3000, 2000. Fotografie, 285,5 x $350 \mathrm{~cm}$. museum kunst palast, Düsseldorf

Kunstwerk zueinander erweckt er den Eindruck, eine spezifische Ateliersituation der Künstler bei der Arbeit an seinem Werk - wiederzugeben und damit szenischnarrativ strukturiert zu sein. Daher sei zunächst eine Lektüre in diesem Sinne versucht. ${ }^{8}$

Von einem innerbildlich nicht eindeutig lokalisierbaren, offensichtlich ein wenig erhöht gelegenen Blickpunkt aus erhalten wir Einblick in das hell erleuchtete Atelier eines Künstlers, das sich, wie die Raumschräge suggeriert, in einem Dachgeschoß befindet. Unmittelbar vor und unter unserem fiktiven Standort gibt es eine Treppe, doch ist der eigentliche Zugang in das Atelier durch ein labil anmutendes Gerüst behindert. Es bildet die Auflagefläche für einige schmale, unregelmäßig verlegte Bretter. Im Atelier befindet sich allerlei Mobiliar: Gerüste mit Holzplanken, Ablagetische und -schränke, ein Stuhl sowie eine durch den Bildrand leicht angeschnittene große Leiter aus Aluminium, deren vordere Standfläche nicht genau lokalisiert werden kann, vermutlich aber mit dem fiktiven Betrachterstandpunkt die erhöht gelegene und wohl auch nicht feste Unterlage teilt. Den Blickfang bildet die

8 Vgl. Zeising (2002), S. $23 f$. 
Skulptur eines hingelagerten nackten Mannes mit erhobenem Arm, die - setzt man ihre Größe ins Verhältnis zur Höhe des Ateliers - als kolossal zu bezeichnen ist. Es gibt keine Spuren einer unmittelbaren Arbeit an ihr, etwa durch ein zur Seite gelegtes Arbeitsgerät oder Dreck am Boden, doch deutet einiges indirekt auf die Arbeit an ihr hin: Denn die Aluminiumfolie auf dem beigestellten Tisch läßt Plastikplanen assoziieren, die in einer Bildhauer-Werkstatt dazu benutzt werden, einen Tonklumpen feucht zu halten, und die Flecken auf dem Oberkörper der Statue suggerieren, daß das Material an diesen Stellen noch nicht durchgetrocknet ist. Außerdem ist sie offenkundig unfertig. Denn sie besitzt nur einen Fuß, und auch der Oberkörper der Figur scheint nur bozziert zu sein - es sei denn, man betrachtet beides als intendiert, und damit das Werk als Torso.

An der Längswand des Ateliers lehnt ein Ölgemälde, das der dominanten Grüntöne wegen wohl als Landschaftsdarstellung zu identifizieren ist. Ob sein non finito ebenfalls beabsichtigt ist oder nur einen kontingenten Zustand in einem Werkprozeß spiegelt, läßt sich schwer entscheiden.

In der rechten oberen Raumecke sehen wir schließlich den jungen Künstler, der bis auf ein Paar Sandalen unbekleidet ist und sein Geschlecht mittels einer Malerpalette vor uns verbirgt. Auf ihr sind einige Farben präpariert, jedoch nicht das Grün des Landschaftsbildes. In der Tat blickt der Künstler auch gar nicht auf das Gemälde, sondern auf die Kolossalstatue, und zwar in einer Haltung, die den Eindruck einer kontrollierend-begutachtenden Schau nach einem Arbeitsgang vermittelt, was mit der Abdeckung des Arbeitsmaterials ja konform geht. Unübersehbar groß hängt im rechten Bildvordergrund eine fotografische Reproduktion der Skulptur in Form eines ausgerissenen Zeitungsartikels. Vom Arbeitsplatz des Künstlers ist er zu weit entfernt, um ihm als bildliche Vorlage zur Ausführung des Werks zu dienen. Er scheint daher für uns externe Betrachter an dieser Stelle arrangiert worden zu sein, was uns die Entzifferung der (unvollständigen) Überschrift: „Im Rascheln der Feigenblä[tter]. Eine Enthüllung und Verhüllung nackter Antik[...] « ermöglicht. Es handelt sich um die Besprechung der mit "Das Feige(n)blatt " betitelten Ausstellung über Nacktheit in der griechischen Plastik, die im Spätsommer des Jahres 2000 in der Münchener Glyptothek stattfand. ${ }^{9}$ An dem Zeitungsausschnitt fallen Rußspuren ins Auge, die auch das fragile Gerüst über der Treppe im Vordergrund aufweist. Daß das Atelier einem schweren Brand ausgesetzt war, suggeriert auch das metallene Treppengeländer, dessen linker Teil zu einem stark verbogenen Stangengewirr zusammengeschmolzen ist. Der übrige Raum scheint von diesem Brand aber nicht in Mitleidenschaft gezogen worden zu sein.

Der Titel des Bildes bestätigt, was der Großteil der Betrachter längst bemerkt haben wird: das in Arbeit befindliche oder soeben beendete Werk ist ein Capolavoro antiker Skulptur: der Barberinische Faun, also jene berühmte hellenistische Groß-

9 Vom 18. Juli bis 29. Oktober 2000; vgl. den Katalog von Peter Prange und Raimund Wünsche, München 2000. 
plastik eines Satyrs in unverhohlener Nacktheit und lasziver Pose, die im frühen 17. Jahrhundert in den Festungsgräben der römischen Engelsburg aufgefunden, von Gian Lorenzo Bernini ergänzt wurde und sich heute in der Münchner Gyptothek befindet. ${ }^{10}$ Es ist das "Bild im Bild «, also der illustrierte Zeitungsausschnitt, der uns anzeigt, daß das vermeintlich in Arbeit befindliche Werk im Atelier bereits Gegenstand einer Kunstausstellung war, und so die Geschehenslogik - wir erwarten in einem Ateliersujet die Anfertigung eines Originals und nicht etwa einer Kopie - aufbricht. Was hier gefertigt wird, kann also nur ein mindestens gleichgroßes, wenn nicht sogar größeres ${ }^{11}$ Derivat des Münchner "Fauns« sein.

Das also nur scheinbar so traditionelle Atelierbild wartet bei eingehender Betrachtung und logischem Nachvollzug seiner Narration mit kalkulierten Brechungen auf. So wird uns ein desaströser Brand suggeriert, der sich gleichwohl auf eine Raumecke beschränkt hat. Vor allem aber ist der Künstler im Bild eigentlich ein Maler, wie seine Palette, das Landschaftsgemälde und eine weitere Leinwand ${ }^{12}$ suggerieren. Er arbeitet hier aber an einer Skulptur. Mit ihr teilt er ebenso die Nacktheit wie die hierdurch aufgerufene Konnotation des Antiken - gleichwohl könnte man sich seinen Körper »antiker" vorstellen, und das sicherlich nicht nur der störenden Sandalen wegen. Und schließlich wird der Faun, bei dem es sich eigentlich um das Produkt eines Bildhauers handelt, anscheinend modelliert. Allerdings deutet sein helles Inkarnat gar nicht auf Ton, sondern auf Marmor oder Gips, also Materialien, die durch das Herausarbeiten einer Form aus einem Block entstehen. ${ }^{13}$ Die Frage "Skulptur oder Plastik", die in einem Atelier, in dem eine Werkgenese inszeniert wird, durchaus von Belang ist, wird durch ein signifikantes Detail wohl doch entschieden: Das Werk ist ein Torso; es besitzt gerade dort eine Fehlstelle, wo sie das Münchner Original nicht hat, denn ihm fehlt der rechte Fuß. Ein Block aus Marmor wäre also unrettbar verschlagen. Nur ein modellierender »Plastiker« kann aus diesem Werk noch den Barberinischen Faun fertigen. ${ }^{14}$

10 Schlafender Satyr, sog. Barberinischer Faun; Marmor; Höhe: 2, 15 m; 2. Hälfte 3. Jahrhundert v. Chr.; vgl. Helas (1999); Walter (1993); Zanker (1998).

11 Geht man davon aus, daß das Atelier auch im hinteren Bereich noch annähernd drei Meter Höhe mißt, müßte es sich um eine leicht vergößerte Variante handeln.

12 Sie ist an das Podest des Künstlers gelehnt und für den Betrachter nur von hinten zu sehen; möglicherweise ist auch der Gegenstand an der Atelier-Rückseite eine monumentale gerahmte Leinwand.

13 Eine begrifflich präzise Unterscheidung der beiden Formen nimmt Michelangelo in seinem Antwort-Brief auf die von Benedetto Varchi initiierte Paragone-Anfrage von 1547 vor: "Io intendo scultura quella che si fa per forza di levare; quella che si fa per via di porre è simile alla pittura (zit. nach: Benedetto Varchi - Vincenzio (1998), S. 84, Hervorh. V.v.R.) Im Englischen wird bekanntlich zwischen "sculpture« and "plastic" unterschieden.

14 Das Original ist eine Marmor-Skulptur, die jedoch gerade am rechten Bein neu ergänzt wurde; vgl. Walter (1993), passim. 
Die Irritationen der Betrachter stellen sich aber nicht nur aufgrund dieser geschehenslogischen Inkonsistenzen ein, sondern auch bei der Wahrnehmung des Ateliers an sich. Es sind die Größen der in ihm verteilten Gegenstände gerade in ihrem Verhältnis zueinander, die jede räumliche Logik unterlaufen: Ließe sich die Monumentalität der Leiter und des Zeitungsausschnitts im Vordergrund noch mit der Nähe dieser Gegenstände zur Kameralinse erklären und die der Skulptur mit dem gestalterischen Willen - oder der Hybris - ihres Schöpfers, so sind der Stuhl und die Steckdose an der Längswand doch eindeutig fehlproportioniert. Es ist also eine manipulierte Welt, die uns Lois Renner hier vor Augen stellt. Zwei Erklärungen sind für diesen Befund möglich: die (nachträgliche) digitale Bearbeitung der Fotografie oder die Manipulation der Gegenstandswelt vor der Kamera und vor dem Fotografieren. Bei Renner trifft letzteres zu: die Werkstatt des Barberinischen Faun ist ein von ihm selbst gebautes und mit ebenfalls eigens gefertigten Gegenständen ausgestattetes Atelier en miniature, das im Maßstab 1:10 die erste Werkstatt des Künstlers in seinem Salzburger Elternhaus nachbildet. ${ }^{15}$

Es ist die als "Fiktionssignal ${ }^{16}$ dienende Steckdose, die, der Funktionsweise eines trompe-l'ceil vergleichbar, den performativen Prozeß aus Täuschung und »EntTäuschung« des Betrachters in Gang setzt. Mit Wissen um den ontologischen Status des Ateliers - also im Zustand der »Ent-täuschung«- klären sich die Verhältnisse, die das Staunen über die künstlerische Leistung auslösen: Die Kolossalstatue entpuppt sich als Miniatur-Faun, das Landschaftsbild als von Renner gemaltes Mini-Bild, der Drehstuhl als Modellmöbel, und auch alle übrigen Gegenstände als vom Künstler gebastelt und gehobelt. Das durch die Pluralität der Gattungen im Atelier angeschlagene Thema künstlerischer Universalität findet seine Entsprechung in der tatsächlichen künstlerischen Vielseitigkeit Renners: Er malt und bastelt, modelliert Skulpturen und baut Welten, die er arrangiert und deren Arrangement er durch die Kamera prüft. Erst in einem letzten Schritt ist er Fotograf, der dann aber den eigentlichen "photographischen Akt" (Dubois), der das Arrangement zum Bild werden läßt, delegieren muß. ${ }^{17}$

\section{Ein allusives Tableau: die »Meniñas» im Atelier}

Das Wissen um die Fiktion der Atelier-Welt erklärt zwar die raumlogischen, aber nicht die geschehenslogischen Inkonsistenzen im Barberinischen Faun. Wie sind die Gattungen in Renners Ateliers inszeniert und was bedeutet das für den medialen Sta-

15 Vgl. Rupert Pfab, in: heute bis jetzt (2002), S. 147.

16 Ein handelt sich um einen von Wolfgang Iser gesprägten Terminus der Erzähltheorie, der sich m. E. auf die Gattung der Modell-Fotografie gut übertragen läßt.

17 In einem im Juni 2002 in der Ausstellung »heute bis jetzt " geführten öffentlichen Gespräch von Lois Renner mit Rubert Pfab berichtete Renner, er habe er eine "zufällig" vorbeigekommene Journalistin gebeten, den Auslöser zu betätigen. 


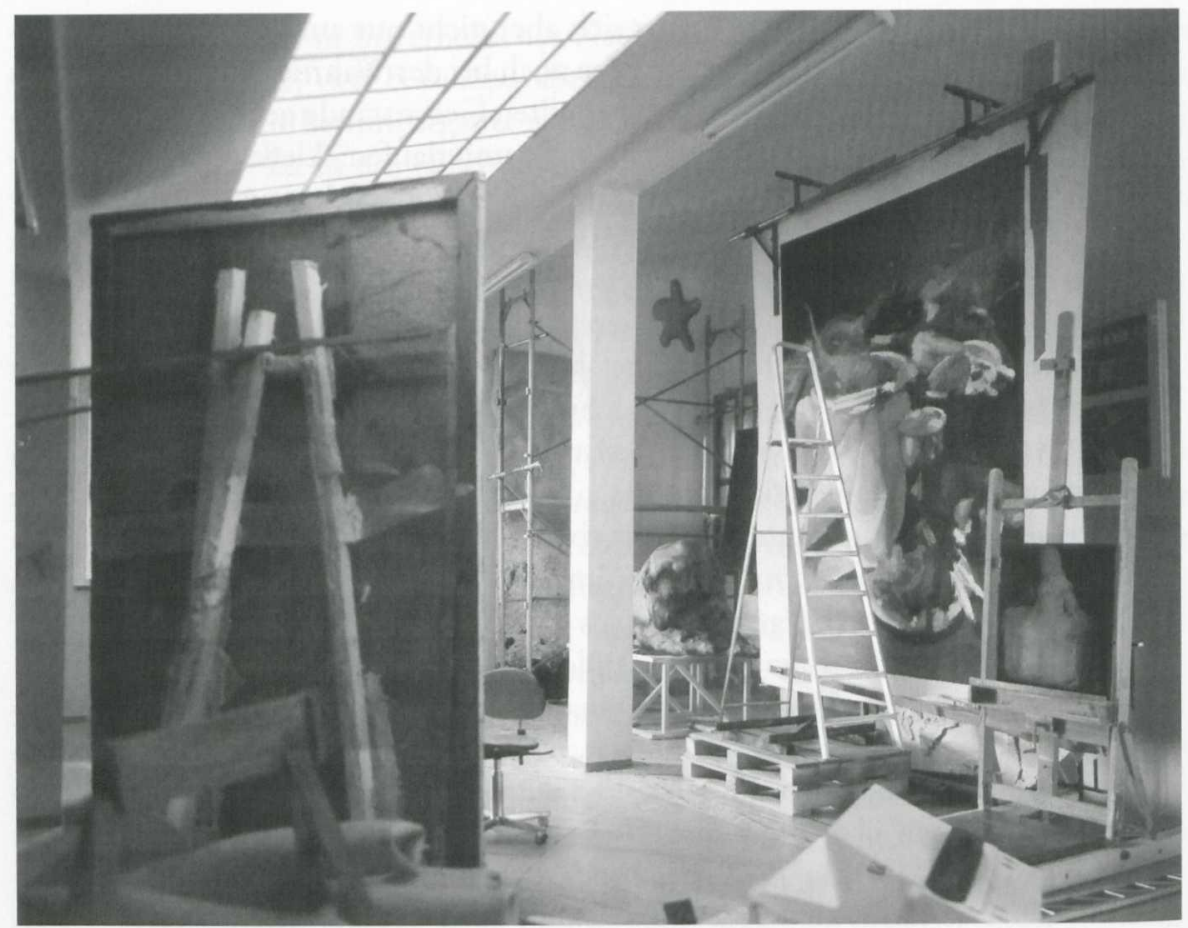

2. Lois Renner, Meniñas, 1992. Fotografie, 152 x 190 cm.

Privatsammlung

tus der Fotografie, die er so gern als Malerei verstanden wissen möchte? Ich möchte diese Fragen unter Einbeziehung eines weiteren Werks beantworten, und zwar der Meniñas $^{18}$ von 1992 (Abb. 2), die den Dialog von Malerei und Fotografie noch auf einer weiteren Ebene inszenieren. Denn dieses Werk rekurriert nicht nur im Titel auf Velázquez' Inkunabel neuzeitlicher Atelierdarstellungen im Typus des Künstlers bei der Arbeit an seinem Werk, ${ }^{19}$ auch die Raumaufteilung verweist auf Velázquez' Arbeitsraum, und zwar durch die Übernahme der monumentalen Leinwand im linken Bildvordergrund, die uns Velázquez nur in Rückansicht präsentiert und von der wir bekanntlich nicht wissen, was für ein Bild sie trägt. „Woran arbeitet Velázquez? « lautet die Frage, um deren Beantwortung sich die kunsthistorische Forschung bekannt-

$18152 \times 190 \mathrm{~cm}$; Privatsammlung Düsseldorf. Eine sehr ähnliche Fassung mit dem Titel Zwerge existiert in Wiener Privatbesitz; Farbabbildungen in: Lois Renner (2003), S. 57 und 60 .

19 Vgl. das Kapitel 8 »Bilder vom Maler/ Bilder vom Malen«, in: Stoichita (1998), S. 224298, speziell zu den Meniñas, S. 278-286; ders. (1986), S. 165-189; allg. zur Gattung: Kleffmann (2000). 
lich immer wieder bemüht hat, die aber implizit voraussetzt, ${ }^{20}$ daß das Bild überhaupt als narratives Tableau zu lesen ist, und damit der Künstler, das Kunstwerk sowie die Personen im Atelier und im Spiegelbild in einem raum- und geschehenslogischen Verhältnis zueinander stehen. ${ }^{21}$ Doch läßt sich die Frage heuristisch auf Renners Produktionsszenarien, in denen der Verweis auf Velázquez' enigmatisches Werk sicherlich nicht zufällig erfolgt, übertragen: Woran also arbeitet Renner?

Auch seine Meniñas geben Einblick in ein Künstleratelier, das, anders als das des Barberinischen Faun, nun primär Bilder beherbergt: Neben einer großen Leinwand, die fast die Hälfte der linken Bildfläche einnimmt, sind mehrere Werke zu erkennen: ein ebenfalls monumentales Stilleben, neben ihm eine kleine Tafel auf einer Staffelei mit der Darstellung eines Mädchens sowie ein aufgebockter Wachsblock, ${ }^{22}$ dessen Form an einen kauernden Hund erinnert, hinter diesem ein weitgehend verdecktes abstraktes Gemälde in Weiß-, Gelb- und Grüntönen. Auch an diesen Werken wird offenbar noch gearbeitet. ${ }^{23}$ Allerdings fallen bei eingehenderer Betrachtung wieder zahlreiche raum- und geschehenslogische Inkonsistenzen ins Auge, die diesen Eindruck sukzessive in Frage stellen. So suggeriert das hochformatige Stilleben zwar durch sein Sujet, ein Ölgemälde zu sein, ${ }^{24}$ doch irritiert seine Befestigung auf einer weißen Projektionsfläche statt auf einer Staffelei. Auf einer Staffelei befindet sich hingegen das Bild mit der Silhouette eines Mädchens, in dem man die Infantin Margarita aus Velázquez' Gemälde wiedererkennt. Durch die ungewöhnliche Positionierung der Figur im Bildfeld will man es für eine Studie (zum großen Leinwandgemälde mit den Meniñas?) halten, doch handelt es sich offensichtlich um eine Fotografie, ${ }^{25}$ was weder mit dem non finito- Zustand der Figur

20 Foucault (1995), S. 33 (mit Bezug auf die Leinwand): »Hartnäckig unseren Blicken entzogen, verhindert sie, daß die Beziehung der Blicke jemals feststellbar ist und definitiv hergestellt werden kann".

21 Vgl. den Literaturüberblick in Kesser (2000).

22 So Lois Renner in einer an mich gerichteten Email vom 17.11. 2002; modelliert hat ihn der ebenfalls aus Österrreich stammende, in Köln lebende Maler Siegfried Anzinger.

23 Dies ist auch deswegen wichtig, weil es sich bei Bildern im Atelier grundsätzlich ja auch um Reproduktionen von Werken handeln könnte, die der Künstler als Vorbild oder Anregung benutzt, was das Thema des künstlerischen Kanons aufrufen würde. Auch dafür gibt es in Renners Oeuvre Beispiele; ich erwähne nur Flora und Zephyr von 2001 in der Sammlung Essl in Klosterneuburg (vgl. Lois Renner 2003, S. 281) sowie P.P.R. 1000 von 1999 im Besitz der GSWB-Bank (ebd., S. 181).

24 Ich kann für das Gemälde kein Vorbild benennen, vermute auch, daß es keines gibt, wofür m. E. das Hochformat und die Kombination von Auf- und Untersicht sprechen. Es wird von Renner in einer jüngeren Arbeit von 2002 mit dem Titel Hunger wieder aufgegriffen, und es taucht auch in der Zusammenstellung seiner "Minibilder«, einer Art persönlichem Bilder-Atlas mit dem Titel Petersburger Hängung wieder auf; siehe die Farbabbildung in: Lois Renner (2003), S. 292.

25 Nach Auskunft des Künstlers handelt es sich um die verkleinerte Fotografie eines Gemäldes nach Velázquez’ Bild. 
vereinbar ist, noch die Positionierung des Werks auf einer Staffelei sinnvoll macht. Nur der Block ist eindeutig das, was er ist. Er hat ebenso wie die "Margarita" auf der Staffelei ein seitenverkehrtes Vorbild in Velázquez' Meniñas, und zwar den vom kleinen Nicolás de Pertusato geneckten Hund, der hier jedoch noch in Form gebracht werden muß.

Begleitet werden diese Darstellungs- und Gattungsbrüche von einigen witzigen Details: So lädt die Haushaltsleiter, die wohl eher selten zum Inventar eines Künstlerateliers gehört, in ihrer prekären Position auf zwei Transportpaletten nicht wirklich zur Nutzung ein, ${ }^{26}$ ferner ist das Podest der Skulptur zu fragil und die Staffelei zu klein geraten, als daß an ihr bequem gearbeitet werden könnte. Renner spielt also nicht nur mit den Gattungen, er verändert auch die Größen der Gegenstände willkürlich und setzt so die Welt - oder die Velázquez'sche Bildwelt - in neue Verhältnisse: Die Infantin wird winzig, ihr Hund groß und das Stilleben monumental - was es $\mathrm{m}$. W. in der neuzeitlichen Bildtradition dieser Gattung, in der die Früchte lebensecht und damit lebensgroß wiedergegeben werden, nicht gibt. Aber - bedenkt man Genese und Status seiner Atelierwelt - ist die Monumentalität der Renner'schen Früchte ja auch nur eine scheinbare. ${ }^{27}$

Wie Velázquez in seinem »tableau-fetiche ${ }^{28}$ ein Produktionsszenario entwirft, in dem die Bezugsgrößen des Ateliers gerade nicht in ein logisches Verhältnis zueinander treten, so tut dies auch Renner, wenn auch natürlich unter anderen Prämissen und mit anderen Implikationen. Dabei kann eine markante Abweichung von Velázquez' Gemälde zur Beantwortung der Frage, wie Renner den Dialog der Gattungen Fotografie und Malerei betreibt, überleiten: Ist es in Velázquez Meniñas das Bildnis des malenden Künstlers, um das herum das "Spiel der Repräsentationen" auf der Sujet- wie auf der übertragenen Ebene inszeniert wird, ${ }^{29}$ so ist an der analogen Stelle in Renners Werk der leere Drehstuhl umso augenfälliger: Der Künstler "malt" zwar dieses Bild, doch macht er (nun) ein anderes, das uns seine Präsenz im Atelier entzieht, weil es seine Anwesenheit vor der Kamera erfordert. ${ }^{30}$

26 Die Haushaltsleiter aus Aluminium ist so etwas wie ein "Markenzeichen" der Renner'schen Ateliers.

27 Ohne den Vergleich mit den Meniñas und seine Implikationen überstrapazieren zu wollen, ist m. E. doch interessant, daß das Thema der Größen der Figuren in ihrer Relation zueinander auch in Velázquez' Gemälde eine Rolle spielt; vgl. hierzu Stoichita (1986).

28 Victor I. Stoichita, Anna Maria Coderch (2003).

29 Vgl. Stoichita (1998), S. 298: „Beide [Velázquez und Vermeer in der Malkunst] situieren ihr Tun im Zentrum des Darstellungsproblems".

30 Auch Velázquez benötigt natürlich ein Hilfsmittel wie den Spiegel, um seine Anwesenheit im Atelier zu erzeugen. 


\section{Der Paragone zwischen Malerei und Fotografie - Ironie als Modus}

Renners »Repräsentationsspiele« sind kein mehr oder weder beliebiges Anzitieren und Dekonstruieren des künstlerischen Kanons, wie man es oft als postmodernen Habitus apostrophiert; sie sind vielmehr "Versuchsanordnungen «, welche die medialen Bedingungen der neuen "Hochkunst« der Fotografie ausloten. Renner betreibt dieses Ausloten im Modus der Ironie, die nicht nur Erkenntnismedium, sondern zugleich auch epistemologisches Äquivalent der paradoxen Bildlichkeit der Fotografie ist. Denn es sind ja gerade die technischen Möglichkeiten, welche seit den achtziger Jahren die neue Ästhetik und Bildwürdigkeit der Fotografie generieren, und zwar die Perfektionierung der von Renner verwendeten "Plattenkamera» sowie die Möglichkeit der Produktion monumentaler Bildgrößen im "XL«-Format in starker Farbigkeit und hoher Auflösung. ${ }^{31}$ Es liegt nahe - und Renner tut das unter den Fotografen seiner Generation auch nicht als einziger $-{ }^{32}$ dieses Ausloten der medialen Bedingungen und Möglichkeiten, das er selbst als "Formforschung" bezeichnet, ${ }^{33}$ über den Rekurs auf die Malerei und damit über den Rückgriff das malte Tableau" zu betreiben. Bevor ich dieses Vorgehen an seinen Werken genauer betrachte, ist vorab eine biographische Komponente zu erwähnen, die in den Werken indirekt und direkt thematisch wird. Der Künstler ist nämlich kein ausgebildeter Fotograf, sondern Maler. Er studierte zunächst am Mozarteum in Salzburg und wechselte 1985 an die Rheinische Akademie in Düsseldorf, wo er u.a. die Klasse von Gerhard Richter besuchte. ${ }^{34}$ Die Gemälde in seinen arrangierten Ateliers sind

31 XL Photography lautet der Titel einer Publikation der Sammlung der Neuen Börse in Frankfurt-Hausen (XL Photography 2000) mit Arbeiten von Andreas Gursky, Candida Höfer, Martin Liebscher, Thomas Ruff u. a. Die Produktion solcher Formate ist überhaupt erst seit den späten achtziger Jahren, in denen Lois Renner anfing zu fotografieren, möglich.

32 Dies gilt sowohl für solche Künstler, die ihre Bilder konstruieren oder inszenieren (siehe hierfür: Das konstruierte Bild (1989); zum Dialog mit der Malerei: Unschärferelation [2000]), als auch für solche, die ihren Arbeiten den Gestus des Dokumentarischen verleihen wie die Fotografen aus dem Umkreis von Bernd und Hilla Becher; siehe hierfür ebd. sowie Gronert (1999), S. 12-31. Allgemein zum Dialog zwischen Malerei und Fotografie: Billeter (1977); Wolfgang Kemp, Theorie der Fotografie 1839-1912, in: ders., von Amelunxen (1980), S. 13-45, bes. 13-24; Diskurse der Bilder (1993); mit Bezug auf die (großformatige) Fotografie des letzten Jahrzehnts: Gronert (2002), S. 37-72, bes. S. 52-65 (mit weiterer Literatur).

33 Im Gespräch mit Silvia Eiblmayr, in: Lois Renner (1995), S. 3-6, hier 3.

34 Nur anmerken möchte ich, daß diese Schülerschaft auch vor dem Hintergrund von Richters Thematisierung der beiden Medien mittels Adaption und malerischen Bearbeitung von Fotografien in seinen sog. "Foto-Gemälden« interessant ist; vgl. zu Richters Verfahren und seinen konzeptuellen Implikationen Misterek-Plagge (1992), sowie Gronert (2002), bes. 39-48. In einer jüngeren Arbeit mit dem sprechenden Titel Richter von (2002), die einen sitzenden Frauenakt in der Ästhetik von Richters Werken zeigt, rekurriert Renner darauf - nun wieder im Medium der Fotografie. Bezeichnenderweise ist es 
also eigene Arbeiten, ${ }^{35}$ weshalb die Modelle in gewisser Weise tatsächlich seine Künstlerateliers sind. Den radikalen Wechsel hin zur Fotografie - von solchen Mini-Bildern für die Modellateliers abgesehen, arbeitet Renner seitdem nicht mehr in diesem Medium - vollzog Renner im Jahre 1991 seiner rhetorischen Stilisierung zum »Maler-Fürsten« zum Trotz. ${ }^{36}$

Gerade vor diesem biographischen Hintergrund ist interessant, daß Renners Fotografien zahlreiche medienspezifische Eigenheiten zu negieren vorgeben oder auch tatsächlich negieren: $\mathrm{Da}$ ist zunächst das schon angesprochene Format der $\mathrm{Ar}$ beiten zu nennen: mit 152 x $190 \mathrm{~cm}$ bzw. 285,5 x $350 \mathrm{~cm}$ haben die Meniñas und der Barberinische Faun im Prinzip die Formate von Gemälden. Wie angedeutet, besteht die paradoxe Komponente dieses Umstands darin, daß es eben gerade technische Möglichkeiten sind, die der Fotografie einen der Malerei ähnlichen TableauCharakter verleihen. Weiterhin behandelt Renner - was sehr selten unter zeitgenössischen Fotografen ist -, seine Arbeiten als Unikate und garantiert, zu keinem Zeitpunkt einen weiteren Abzug vom Negativ in seinem Besitz zu erstellen. ${ }^{37}$ Gerade das, was Fotografie ausmacht, nämlich ihre vergleichsweise leichte und vor allem potentiell unendliche Reproduzierbarkeit, schaltet er dadurch aus. Eine weitere genuine Eigenschaft der Fotografie ist ihre Unmittelbarkeit und Mechanizität, die im berühmten Werbespruch der Firma Kodak "Sie fotografieren und alles andere machen wir" auf den Punkt gebracht wird. Sie wird von Fotografen, die mit arrangierten Bildern arbeiten oder eben gar Modelle fertigen, per se unterlaufen; Renner

die einzige Schwarz-Weiß-Arbeit bislang in seinem Euvre, und sie hat mit 151 x $98 \mathrm{~cm}$ ein vergleichsweise kleines Format.

35 Im Interview mit Hochdörfer (in: La casa, il corpo, il cuore 1999), S. 160 spricht er von seiner »Vorliebe für kleine Bilder".

36 Zitiert seien einige originelle Aussagen Renners zu diesem Thema; zunächst aus dem Gespräch mit Silvia Eiblmayr (in: Lois Renner 1995, S. 3f.): „Wenn man, so wie ich, gerne und viel arbeitet, dann ist es sehr wichtig, daß man es mit schönen Sachen zu tun hat, daß man es lange aushält im Atelier. Und an der Stelle hab ich am meisten Glück gehabt, weil ich mich ja den ganzen Tag mit dem allerschönsten Zeug beschäftigen kann, das ein anderer Künstler in seinem Atelier nicht mehr benützen sollte. Ich denke da an große Formate, an gegenständliche Malerei, an ungegenständliche Malerei - an all diese Dinge. Dinge, die ich ja eigentlich mag. Denn vor meiner Hochschulzeit hab ich ja nicht gewußt, daß es moderne Kunst gibt. Ich dachte, ich könnte Maler werden und ein wahnsinnig schönes Bild malen, mehr nicht. Und als dann all meine Hoffnungen so brutal zerstört wurden in mir, durch die strenge Hochschule zum Glück, habe ich nach diesem frühen 'Malertod' anfangen können mit umgekehrten Vorzeichen alles wieder hervorzuholen" und ebd., S. 4f: "Anders als die in den fünfziger-Jahren geborenen Künstler habe ich den letzten Zipfel der Malerei nicht mehr erwischt. Deshalb habe ich mich gleich dagegengestellt. Deshalb mußte ich sofort ein radikales Malmanöver einleiten, die Zeit war genau richtig dafür. Keine Keilrahmen einkaufen gehen, keine Leinwände aufspannen, keine Hartware mehr herstellen.«; S. 5: „Und über Nacht war ich mir ganz sicher, daß es für mich ein Leben nach dem 'Malertod' gibt."

37 Vgl. hierfür Lois Renner (2003), S. 28. 


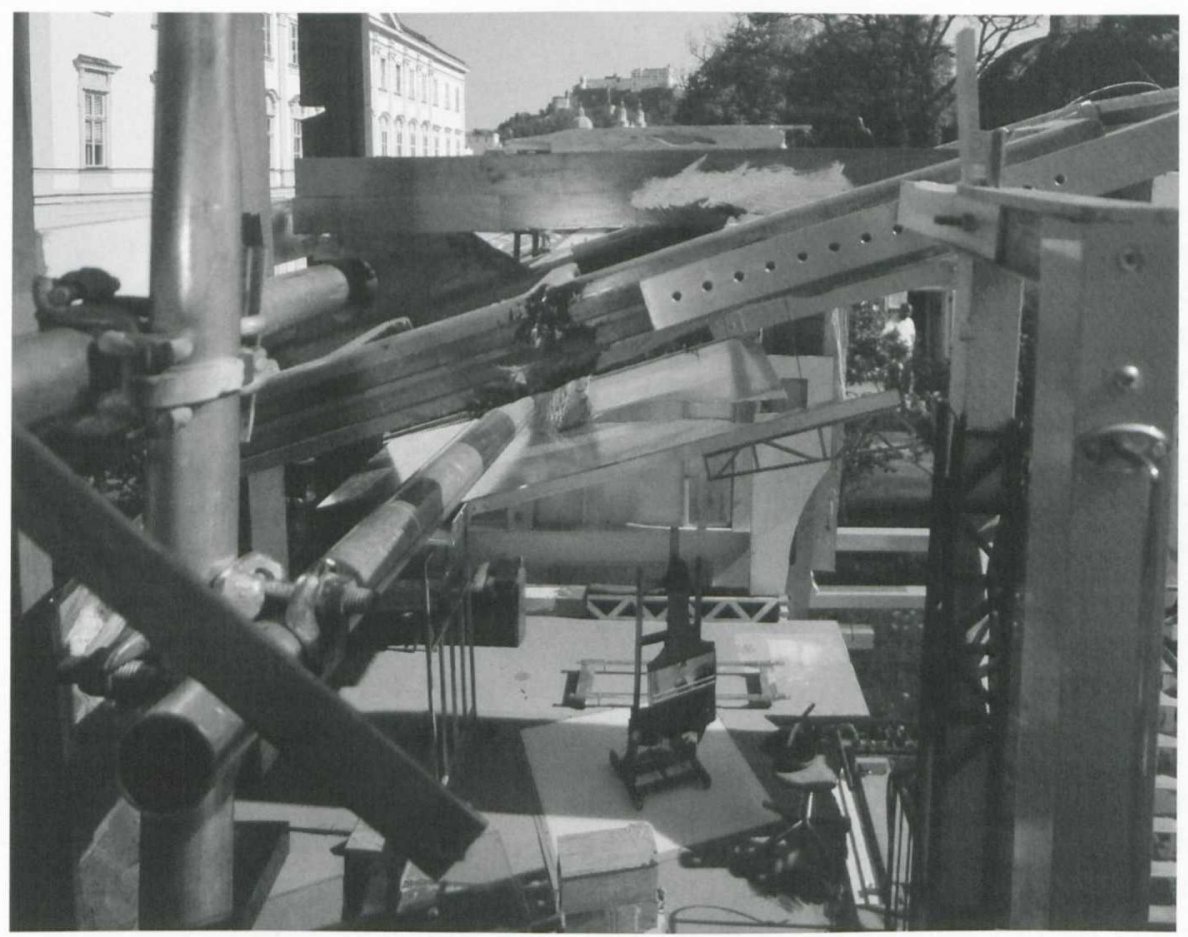

3. Lois Renner, Mirabellgarten, 1999. Fotografie, 152 x $190 \mathrm{~cm}$. Privatsammlung

forciert dies noch, wenn er dabei innerbildlich die handwerkliche Komponente des "Bildermachens« herausstellt. Denn nicht nur verweisen die zahlreichen Gerüste, die in seinen Modellen herumliegen, aber nicht durch die Geschehenslogik der Sujets erforderlich sind, metaphorisch auf diese zeitaufwendige und akribische "Arbeit« am Bild, er konnotiert auch die Tätigkeit des Malers im Atelier handwerklich und hebt hierdurch auf die Doppelbedeutung von "Malen" ab. So steht er in Klass/Super ${ }^{38}$ von 1991, das er rückwirkend als sein erstes Werk bezeichnet, hinter einer großen Lache ausgekippter Farbe industrieller Fertigung und trägt einen selbstgebastelten Zeitungshut, in Das Schaf $(1991)^{39}$ ist eine für das Anstreichen von Wänden benutzte Farbwalze im Atelier "monumental" aufgebockt, im Zentrum von Mirabellgarten (1999; Abb. 3$)^{40}$ liegt ein breiter Pinsel, wie er für das Auftragen von Lackfarbe benutzt wird, und in Lindgrünes Baugerüst (1992; Abb. 4) ${ }^{41}$ stehen zwei große bemalte Holztafeln, bei denen es sich um abstrakte Gemälde zu

$38225 \times 180 \mathrm{~cm}$; Privatsammlung Wien; siehe ebd., S. 32. Die Selbstbildnis ist eine verkleinerte, im "Atelier« aufgestellte Ganzkörper-Fotografie.

39152 x $190 \mathrm{~cm}$; Sammlung Josef Neuhauser, Salzburg; siehe ebd., S. 33.

40152 x $190 \mathrm{~cm}$; im Besitz des Künstlers; Farbabbildung ebd., S. 193.

41152 x $190 \mathrm{~cm}$; Privatsammlung; Farbabbildung ebd., S. 61. 


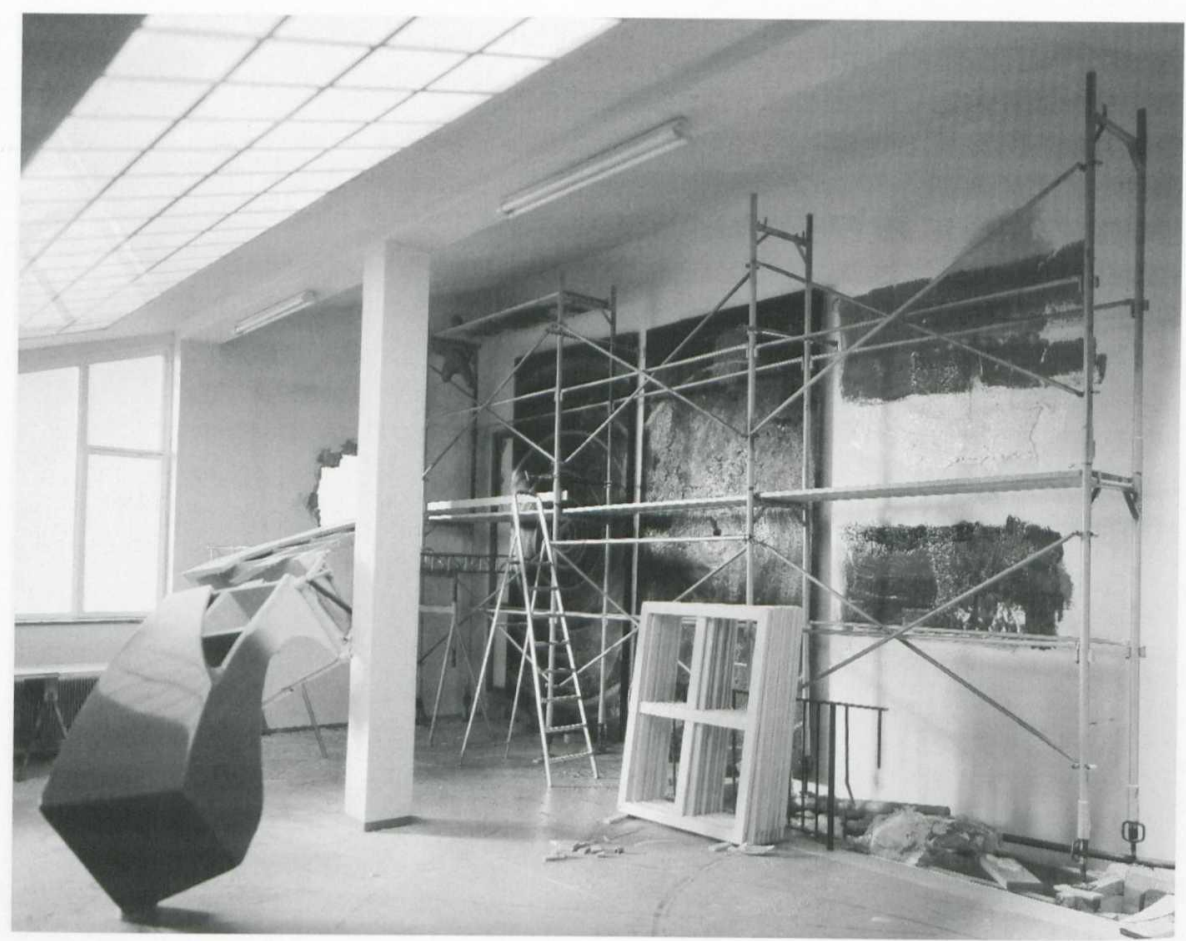

4. Lois Renner, Lindgrünes Baugerüst, 1992. Fotografie, 152 x $190 \mathrm{~cm}$.

Privatsammlung

handeln scheint, neben einem unmittelbar auf die Wand applizierten "Streifenbild" in Arbeit. Dabei wecken das Fehlen des Bildträgers, der Bauschutt auf dem Boden und ein davor stehendes Gerüst beim Betrachter Zweifel über den Status dieses Bildes. Auch hier ist ein biographischer Umstand zu erwähnen: Renners Eltern führen in Salzburg einen Handwerksbetrieb, und er selbst absolvierte vor dem Studium der Malerei eine Malerlehre, die er mit dem Meistertitel abschloß. Die in mehreren Arbeiten integrierten Aufschriften Malerei Renner ${ }^{42}-$ der Name des väterlichen Betriebs - haben also Doppelsinn.

Mechanizität und Unmittelbarkeit des Mediums werden von Renner jedoch nicht durchgängig negiert, sondern gelegentlich sogar pointiert. So fixiert er in $3 \mathrm{Uhr}$ $14^{43}$ mittels einer Uhr im Vordergrund den genauen Zeitpunkt der Aufnahme des

42 Beispielweise in Barberinischer Faun II (2002), der auf der Einladungskarte zur Ausstellung "Augenblick. Foto/Kunst" der Sammlung Essl von 2002 abgebildet war.

$43225 \times 180$ cm; 1995; Privatbesitz. Farbabbildung ebd., S. 93. Es gibt eine schöne Bemerkung Renners über die Zeitlichkeit der Medien: "Auch wenn es eigentlich ihre Aufgabe ist, Zeit zu negieren, so hat die Malerei für mich eine lange Zeit. Die Fotografie eine kur- 
Fotos, und damit seine Genese im Bruchteil einer Sekunde. ${ }^{44}$ Wenn er sich im Barberinischen Faun ins Bild begibt und den Akt des Auslösens an einen Dritten delegiert, in den Meniñas jedoch ostentativ verschwunden ist, um das eigentliche Bild anzufertigen, macht er den paradoxalen Dualismus der Fotografie zwischen ihrer Mechanizität und Objektivität einerseits und ihrer Subjektivität anderseits ${ }^{45}$ geradezu spielerisch thematisch. Die dem Medium eigene Subjektivität pointiert er wiederum, wenn er nicht nur Referenzen an seinen Ausbildungsgang ins Bild nimmt und in den Modellen sein erstes Atelier nachbaut, sondern in zahlreichen Werken auch seinen Namen unterbringt (vgl. Abb. 7) und damit anzeigt, in wessen Welt wir blicken. Die originellste Variante begegnet in einem bislang unerwähnten Detail im Barberinischen Faun: Die zu einem Knäuel zusammengeschmolzenen Stangen des Gitters im Vordergrund bilden die beiden ersten Buchstaben seines Namens - eine Signatur, die an die Signier- oder Monogrammierform eines walten Tableaus« erinnert.

\section{Das Atelier als Bühne, die Schöpfung als Spiel}

Obwohl das Bauen von Modellen zum Anfertigen von Fotografien der topischen Unmittelbarkeit der Fotografie im Prinzip natürlich zuwiderläuft, so macht es doch nur in Verbindung mit diesem künstlerischen Medium überhaupt Sinn. Der Grund dafür liegt in der Illusionskraft und zugleich der Realitätsmacht der Fotografie, ihrer - paradoxerweise durch ihre automatische Genese bedingten "Glaubwürdigkeit« ${ }^{46}$ die für das von Renner betriebene Spiel der Repräsentationen und Proportionen unabdingbar ist. Gerade vor dem Hintergrund der technischen Entwicklung der Gattung ist dies interessant, denn das Arbeiten mit analog erzeugten Bildern ist ja, wenn man so will, bereits ein "antiquierter" Umgang mit dem Medium der Fotografie. Es mutet fast ein wenig kurios an, daß in einer Zeit, in der die Technik der digitalen Bildbearbeitung die vergleichsweise mühelose Si-

ze und das Modell gar keine. Bildlich gesprochen muß das Modell sehr lange ruhig warten, die Malerei beeilt sich und die Fotografie kennt überhaupt nur den Augenblick « (im Gespräch mit Achim Hochdörfer [in: La casa, il corpo, il cuore 1999], S. 160).

44 Cartier Bresson emfpand die »Poetik des Augenblicks" als die Essenz des fotografischen Bildes; vgl. hierfür Stephan Berg, Zur Einführung, in: Unschärferelation (2000), S. 812 , hier 10.

45 Vgl. Groys (1997), S. 127-144 und Bazin (1975), demzufolge sich Fotografie »in Abwesenheit des Menschen"vollziehe (S. 24).

46 Bazin (1975), S. 23; vgl. Berg, Zur Einführung, in: Unschärferelation (2000), S. 8: „Wir glauben fotografischen Bilder auch dann noch, wenn wir wissen, dass sie lügen können, weil die Mechanik ihrer Bilderzeugung eine bestimmte Gewähr für die Wahrheit des Abgebildeten in sich zu tragen scheint. Darin steht das Medium in krassem Gegensatz zur Malerei, in der jeder Pinselstrich, geradezu ein Beweis für die subjektive Setzung ist, weil er der Malhand entspringt." 


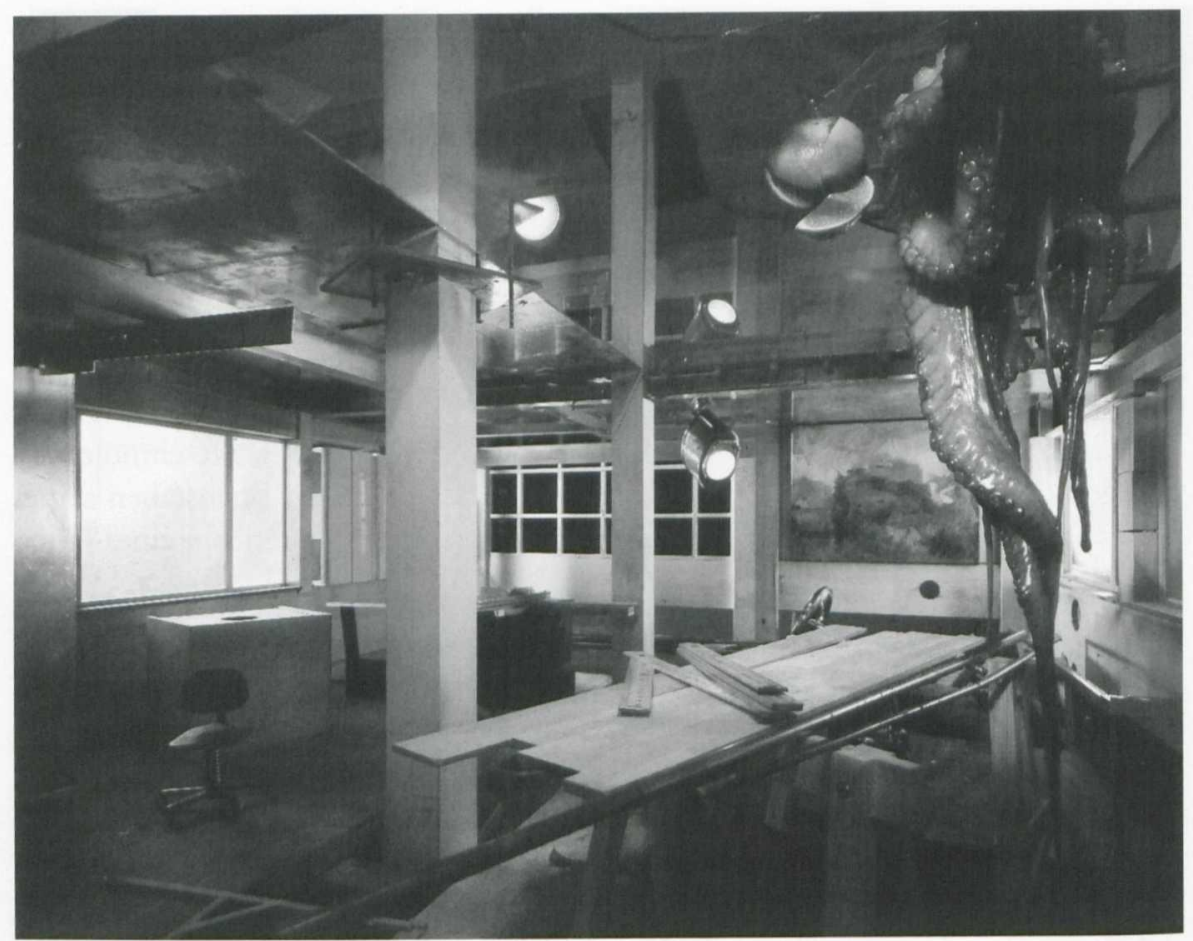

5. Lois Renner, Parterre, 2001. Fotografie, $120 \times 150 \mathrm{~cm}$. Privatsammlung, Düsseldorf

mulation von »Wirklichkeiten « ermöglicht, ${ }^{47}$ Künstler wie Thomas Demand, Edwin Zwakman, Michael Boberg und Lois Renner ${ }^{48}$ ihre Kulissen in aufwendigen Verfahren basteln, und dies teilweise so perfekt, daß die Täuschung über ihren ontologischen Status beim Betrachter mitunter lang anhält. ${ }^{49}$ Doch anders als in der digitalen und computergenerierten Fotografie, bei der das konzeptuelle Moment ja genau darin besteht, den "Schritt in die Virtualität, vom Weltbild zur Bildwelt " 50 zu verwischen, wird in den Arbeiten dieser »Modellbauer« die Fiktion bei intensi-

47 Auch auf diese Form der Bilderzeugung scheint Renner ironisch anszuspielen, wenn er in jüngeren Arbeiten im Vordergrund einen Computer samt einer vom Bildrand überschnittenen Tastatur einfügt wie in Workstation 1000 aus dem Jahr 2000 (225 x 180 $\mathrm{cm}$ ).

48 Ich nenne nur jene Künstler, die in etwa einer Generation zuzurechnen sind; es handelt sich selbstverständlich nicht um eine Künstlergruppe im eigentlichen Sinne des Wortes.

49 Dies gilt gerade für die Meniñas; nur in der leicht divergierdenden Wiener Fassung gibt es ein veritables Fiktionssignal in Form einer Büroklammer auf dem Holzbock im Vordergrund.

50 Von Brauchitsch (2002), S. 253. 


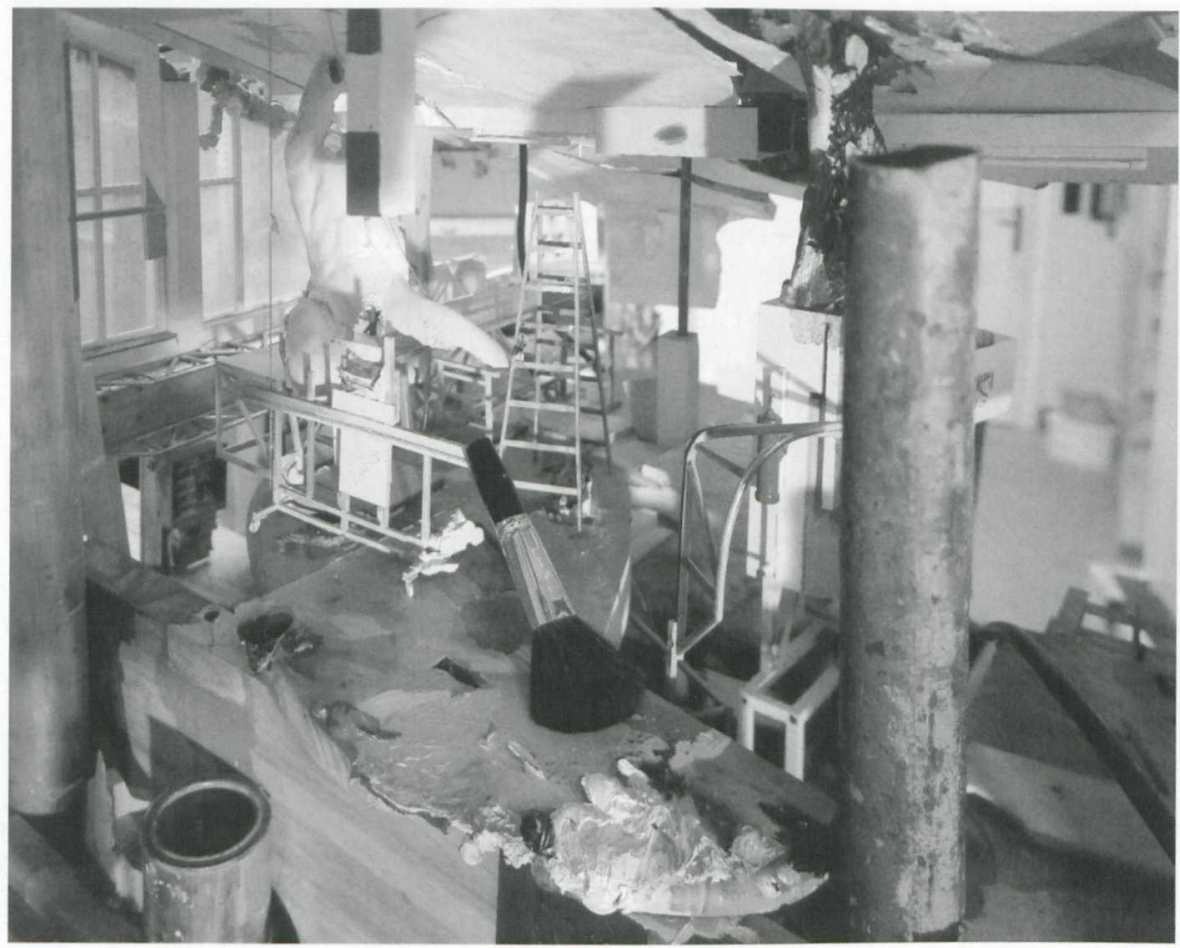

6. Lois Renner, Der Faun im Atelier, 1997. Fotografie, 180 x $225 \mathrm{~cm}$. Hessische Landesbank, Frankfurt a.M

verer Betrachtung der Bilder früher oder später durchschaubar und damit thematisch. Renner erzeugt diese "Ent-Täuschung des Betrachters mittels der oben als "Fiktionssignale« bezeichneten Indikatoren der Artifizialität, die er spielerisch variiert: So sind die Steckdose im Barberinischen Faun (Abb.1) oder das Bic-Feuerzeug in Gerüst $t^{51}$ veritable Fiktionssignale, weil dem Betrachter ihre Größe in Relation zu anderen Objekten der Dingwelt bewußt ist. Weil er metaphorisch auf die Maßverhältnisse verweist, hat der im Bildvordergrund von Atelier (Parterre) (Abb. 5) ${ }^{52}$ abgelegte aufgefächerte Zollstock geradezu selbstbezüglichen Charakter. Die Palette mit Schminkpinsel in Der Faun im Atelier (1997; Abb. 6) ${ }^{53}$ spielt auf die originär antike Analogisierung von Farbe und Schminke, und damit als ein lügnerisches,

51120 x 150 cm; 1993; Sei Collection, New York; Farbabbildung in: Lois Renner (2003), S. 68 .

$52120 \times 150 \mathrm{~cm}$; 2001; Privatsammlung Düsseldorf; Farbabbildung ebd., S. 279.

53180 x 225 cm, 1997; Hessische Landesbank, Frankfurt a. M.; Farbabbildung ebd., S. 140 . 
scheinhaftes und unehrliches Element an, ${ }^{54}$ wodurch sie auf der Metaebene auf die Diskrepanz von Schein und Sein verweist. Und schließlich ist auch der an einem Ständer hängende violette Oktopus in Atelier (Parterre) ein veritables Fiktionssignal, wenn auch mit einer ironisch-witzigen Volte: Zwar existiert in der Natur die Krake in verschiedenen Größen, und auch in monumentaler Form - weshalb sie ein doppelt verunsicherndes Moment ins Bild bringt und als Fiktionssignal eigentlich gar nicht taugt -, in der Kombination mit einer Limone erweist sie sich jedoch als eßbar, und damit eben doch als harmloser Tintenfisch.

In jüngeren Arbeiten, wie dem Faun im Atelier (Abb. 6) und Mirabellgarten (Abb. 3), tendiert Renner dazu, den Konstruktcharakter der fingierten Atelierwelt deutlicher zu machen, indem er durch das Fotografieren aus größerer Distanz zum Modell den Bruch der Welten und die die fingierte Welt konstituierenden Gerüste mit ins Bild nimmt. Dabei sucht er diese Arrangements in immer neuen Variationen und aus immer neuen Perspektiven fotografisch zu erfassen. ${ }^{55}$

Letzterer Aspekt ist noch in einem anderen Zusammenhang interessant: Allein durch den Modellbau, insbesondere aber in Verbindung mit dem Sujet des Künstlerateliers ruft Renner in seinen Arbeiten ja einen wichtigen Topos der okzidentalen Denktradition auf, nämlich dem vom Gott als Bildner $-{ }^{56}$ und gerade der Barberinische Faun schlägt das Thema schöpferischer Potenz überdeutlich an. Der Demiurg Renner baut Welten und unterzieht seine Arrangements anschließend einem prüfenden Blick durch die Kameralinse. Wenn er seine Schöpfungen jedoch immer weiter verändert und somit spielerisch als kontingente kenntlich macht, nimmt er auf eine ganz bestimmte Ausprägung dieses Konzepts Bezug, und zwar das vom "deus ludens", vom "spielenden Gott", dessen Kreationen kontingent sind, weil sie eben auch ganz anders hätten aussehen können. ${ }^{57}$ In einer solchen Konzeption, wie sie von Giambattista Marino um 1600 entwickelt wurde, wird die Schöpfung der Welt zum bloßen Scherz des spielenden Gottes, zum »zerstreut-vergnügliche(n) Spiel seines Pinsels.$^{58}$ Sie erzeugt ob ihrer Vielgestaltigkeit Vergnügen und zielt auf die meraviglia des Betrachters, gerade weil sie - und so wendet sich die Kontingenz dialektisch ins Positive - zum Zeichen seines unbegrenzten schöpferischen Vermögens wird.

Obgleich der Rekurs auf das Konzept eines spielenden Gottes von Renner kaum bewußt erfolgt sein wird, scheint mir die Allusion auf ein verwandtes, ebenfalls barockes Denkkonzept doch intendiert zu sein: Ich meine die Welt als theatrum

54 Vgl. von Rosen (2001), S. 430-432; Lichtenstein (1993), S. $38 \mathrm{ff}$. Die loci classici finden sich in den platonischen Dialogen Gorgias (465b), Phaidros (239c-d), Politeia (602c).

55 Vgl. Trummer (1998), S. 134: „Der Vordergund zeigt uns nicht mehr den Estrich eines Ateliers, sondern gleichsam eine Zwischenzone, ein kompliziertes System an Verstrebungen in Bau - oder doch schon das beginnende Äußere des Modells? (mit Bezug auf Duschkabine von 1993).

56 Vgl. zu diesem Topos und seinen Ausprägungen: Curtius (1948), S. 529-531.

57 Regn (2000), S. 359- 382, bes. 371-382.

58 Ebd., S. 380. 


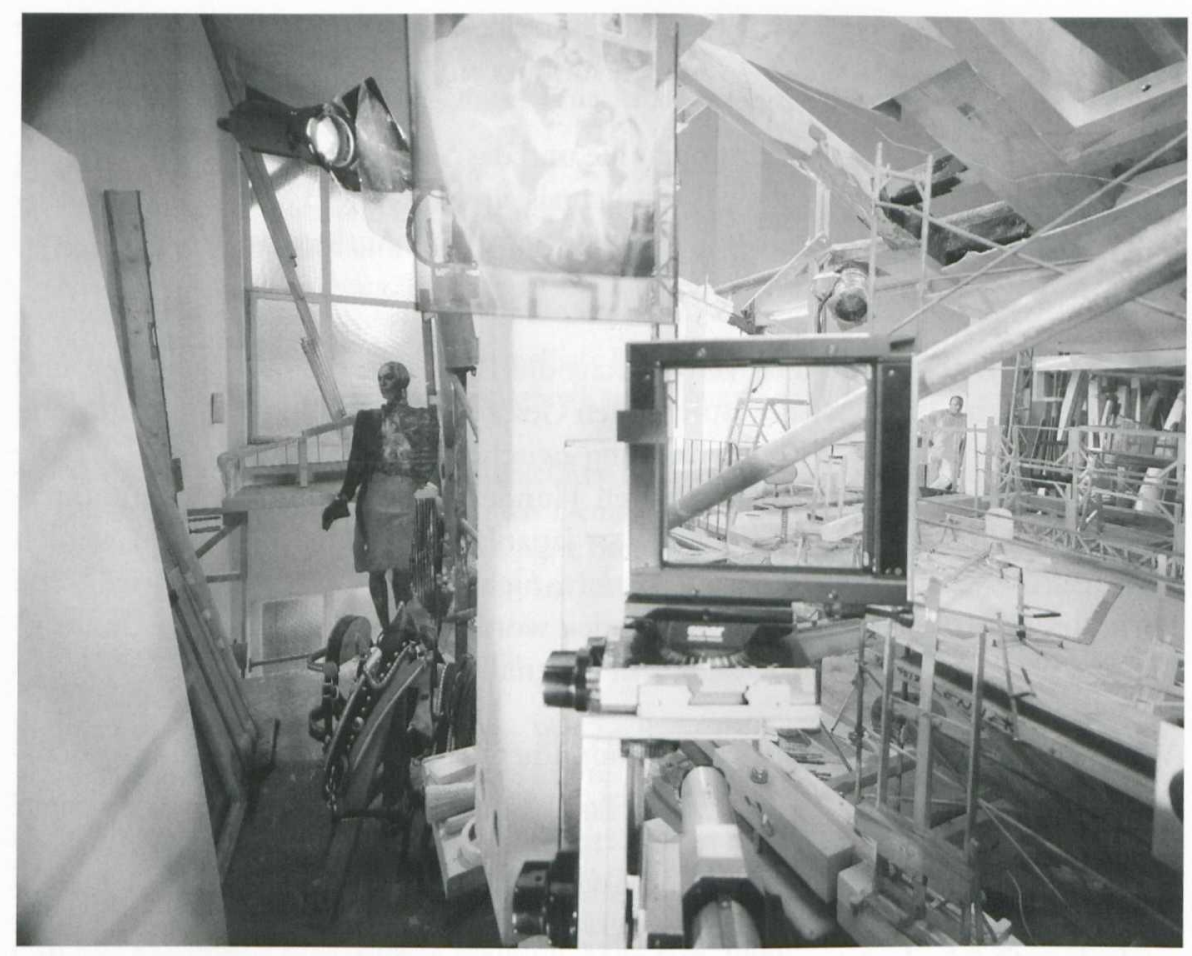

7. Lois Renner, Großes Theater, 1999. Fotografie, $301 \times 360 \mathrm{~cm}$. Sammlung Rheingold, Düsseldorf

mundi, in dem das Leben als Schauspiel gedacht wird, das der Mensch vor Gott als dem Autor und Regisseur der Welt aufführt. Renners bislang größte Arbeit mit den Maßen 301 x 360 cm (1999, Abb. 7), ${ }^{59}$ die einen Einblick ins das reale Künstleratelier bietet, das von einer Schaufensterpuppe »bewohnt« wird, trägt sicherlich kaum zufällig den Titel "Großes Theater«.

So sehr die Beschäftigung mit dem künstlerischen Kanon und die spielerische Dekonstruktion von Meisterwerken als Signum der künstlerischen Tätigkeit der Moderne und zeitgenössischen Kunst gelten können - Renners Rekurse auf "alte" Denkmuster bezüglich der schöpferischen Tätigkeit des Künstlers und Themen wie das Ateliersujet sind gerade in ihrem ironischen, auf die Erkundung ihrer konstitutionellen Bedingungen abzielenden Modus besonders. Wenn sich die Schöpfung des Bildhauers als ein bloßer Abklatsch eines (antiken) Meisterwerks entpuppt, und die Produktion des "neo-spanischen« Malers als mehrfach gebrochene

59 Sammlung Rheingold, Düsseldorf; Farbabbildung in: Lois Renner (2003), S. 203 (mit falscher Maßangabe). 
Bild-Foto-Chimäre, dann schreibt sich Renner darüberhinaus - und einmal mehr in forciert "origineller" Weise - in einen Diskurs ein, der intrinsisch mit der Fotografie als Gattung verknüpft ist.

In ihrem Essay "Über die Photographie und das Simulakrale« von 1984 begreift Rosalind Krauss die Fotografie bekanntlich als ein Mittel der Dekonstruktion zentraler Werte schöpferischer Tätigkeit wie Originalität, Authentizität sowie individuellem Selbstausdruck:

Indem die Photographie die Multiplizität, die Faktizität, die Wiederholung und das Stereotyp im Inneren jeder ästhetischen Geste herausgestellt hat, dekonstruierte sie die Möglichkeiten der Differenzierung zwischen Original und Kopie. [...] Die Praktik des Multiplen, ob man von den Hunderten von grundsätzlich ununterscheidbaren Photographien, die von [...] japanischen Männern gemacht werden könnten - diese Praktik ist von Künstlern nicht nur als niedere oder schlechte Form des ästhetischen Originals verstanden worden. Sie wurde auch als Unterminierung der ganzen Unterscheidung von Original und Kopie aufgefaßt. ${ }^{60}$

Wenn Renner mit der Plattenkamera monumentale, ästhetisch höchst anspruchsvolle und als Unikate auch »originale« einmalige Tableaus schafft, welche die Scheinwelt des Ateliers in einem perfekteren und schärferen Maße wiedergeben, als wir sie selbst mit unserem natürlichen Auge zu erfassen vermögen, arbeitet er wohl an einer "Re-Auratisierung ${ }^{61}$ des fotografischen Bildes, die von einer Sehnsucht nach dem Original getragen ist. Dabei bleibt seinen Bildern das Wissen um ihre technische Reproduzierbarkeit aber nicht nur eingeschrieben, sie bringen es sogar ironisch auf den Punkt - im Gestus des deus artifex.

\section{Literatur}

Ästhetische Grundbegriffe. Historisches Wörterbuch in sieben Bänden, hg. v. Karlheinz Barck u. a., Stuttgart u. a. $2000 \mathrm{ff}$.

Augenblick. Foto/ Kunst (Ausst. Kat. Klosterneuburg 2002), hg. v. Gabriele Bösch, o. O. 2002.

André Bazin, "Die Ontologie des fotografischen Bildes«, in: ders., Was ist Kino?, Köln 1975, S. 21-27.

Boris von Brauchitsch, Kleine Geschichte der Fotografie, Stuttgart 2002.

Ernst Robert Curtius, Europäische Literatur und lateinisches Mittelalter, Bern 1948.

Diskurse der Bilder. Photokünstlerische Reprisen kunsthistorischer Werke (Ausst. Kat. Wien 1993/94), hg. v. W. Seipel, Wien 1993.

60 Krauss (1998), S. 210-223; vgl. auch dies. 2000), bes. S. 217-219 mit Bezug auf die Appropriation-Künstlerin Sherry Levine; Häseler (2002), S. 638-655.

61 Douglas Crimp, zitiert nach Hubertus von Amelunxen, Von der Theorie der Fotografie. 1980-1995, in: Kemp, von Amelunxen (1980-2000), S. 11-22, hier 12. 
Das Feige(n)blatt (Ausst. Kat. München 2000), hg. v. Peter Prange, Raimund Wünsche, München 2000.

Michel Foucault, Ordnung der Dinge. Eine Archäologie der Humanwissenschaften, 13. Aufl., Frankfurt 1995.

Geschichte der österreichischen Kunst, Bd. 6: Kunst des 20. Jahrhunderts, Wien 2001.

Stefan Gronert, "Aussichten. Die Landschaft in der zeitgenössischen Photographie«, in: Insight Out. Landschaft und Interieur als Themen zeitgenössischer Photographie (Ausst. Kat. Innsbruck, Hamburg, Baselland 1999), hg. v. Barbara Hofmann, Zürich, New York 1999, S. 15-18.

-, "Die Bildlichkeit des Abbildes. Die mediale Reflexion der Fotografie bei Gerhard Richter und Jeff Wall«, in: Zeitschrift für Ästhetik und allgemeine Kunstwissenschaft 47, 2002, S. 37-72.

-, "Reality is not totally real. Die Infragestellung des Sichtbaren in der zeitgenössischen Fotografie", in: Grosse Illusionen. Thomas Demand, Andreas Gursky, Edward Ruscha (Ausst. Kat. Bonn, North Miami 1999), hg. v. Stefan Gronert, Köln 1999, S. 12-31.

Boris Groys, Die Logik der Sammlung. Am Ende des musealen Zeitalters, München, Wien 1997.

Jens Häseler, s.v. Original/Originalität, in: Ästhetische Grundbegriffe, Bd. 4, Stuttgart 2002, S. 638-655.

Philine Helas, s. v. Barberinischer Faun, in: Der Neue Pauly. Enzyklopädie der Antike, Bd. 13 Rezeption- und Wissenschaftsgeschichte, Stuttgart 1999, Sp. 389-394.

Heute bis jetzt. Zeitgenössische Fotografie aus Düsseldorf, Teil 2 (Ausst. Kat. Düsseldorf 2002), hg. v. Rupert Pfab, München 2002.

Wolfgang Kemp, Hubertus von Amelunxen (Hg.), Theorie der Fotografie, München 19802000.

Caroline Kesser, Die Meninas von Foucault, München 2000.

Andrea Beate Kleffmann, Atelierdarstellungen im 18. und 19. Jahrhundert, Essen 2000.

Das konstruierte Bild. Fotografie - arrangiert und inszeniert (Ausst. Kat. München 2000), hg. v. Michael Köhler, München 1989.

Rosalind Krauss, "Eine Bemerkung über die Photographie und das Simulakrale", in: dies., Das Photographische. Eine Theorie der Abstände, München 1998, S. 210-223.

-, Die Originalität des Avantgarde-Künstlers und andere Mythen der Moderne, hg. v. Herta Wolf, Amsterdam 2000.

Künstler helfen Alten und Neuen Meistern. Auktion zugunsten der Staatlichen Kunstsammlungen Dresden (Auktionskat. Berlin 2002), hg. v. Achenbach Art Consulting, Düsseldorf 2002.

La casa, il corpo, il cuore. Konstruktionen der Identitäten (Ausst. Kat. Wien 1999), Wien 1999.

Jacqueline Lichtenstein, The Eloquence of Color. Rhetoric and Painting, Berkely 1993.

Lois Renner (Ausst. Kat. Salzburg 1995), Salzburg 1995.

Lois Renner. Luxusbilder (Ausst. Kat. Bregenz 1998), Bregenz 1998.

Lois Renner Bilder/Pictures 1991-2002, hg. von Maribel Königer, Ostfildern/Ruit 2003.

Malerei Renner. Bilder, Fotoarbeiten und Modellskulpturen von Lois Renner (Ausst. Kat. Salzburg 1999), hg. v. Peter Weiermeier, Salzburg u. a. 1999.

Malerei und Photographie im Dialog. Von 1840 bis heute (Ausst. Kat. Zürich 1977), hg. v. Erika Billeter, Bern 1977.

Rainer Metzger, "Tausend Tableaus. Das Ganze und die Unmöglichkeit: Lois Renners Arbeit für Admont", in: Michael Braunsteiner (Hg.), Lois Renner im Stift Admont, Regensburg 2001, S. 20-31. 
Ingrid Misterek-Plagge, Kunst mit Fotografie und die frühen Foto-Gemälde Gerhard Richters, Münster, Hamburg 1992.

Renate Puvogel, »Kultiviertes Chaos«, in: Formalismus. Roland Goeschl, Heimo Zobernig, Lois Renner (Ausst. Kat. Wien 1997/98), Wien 1998, S. 144-160; der Inhalt dieses Beitrags ist in den Renner betreffenden Passagen im wesentlichen identisch mit: dies., "Das Atelier als Thema - Das Atelier als Bild und Raum«, in: Artis Oktober/November 1994, S. 46-51.

Gerhard Regn, "Metaphysische Fundierung und ästhetische Autonomie. Ambivalenzen barocker Kunstkonzeption in Marinos Dicerie sacre«, in: Joachim Küpper, Friedrich Wolfzettel (Hg.), Diskurse des Barock, München 2000, S. 359- 382.

Valeska von Rosen, Mimesis und Selbstbezüglichkeit in Werken Tizians. Studien zum venezianischen Malereidiskurs, Emsdetten/Berlin 2001.

Victor I. Stoichita, "Imago regis. Kunsttheorie und königliches Porträt in den Meninas von Velázquez«, in: Zeitschrift für Kunstgeschichte 49, 1986, S. 165-189.

-, Das selbstbewußte Bild. Vom Ursprung der Metamalerei, München 1998.

-, Anna Maria Coderch, "Goya als Prinzenmacher«, in: Valeska von Rosen u. a. (Hg.), Der stumme Diskurs der Bilder. Reflexionsformen der Ästhetischen in der Kunst der Frühen Neuzeit (Kongreßakten Berlin 2000), Berlin 2003, S. 283-304.

Thomas Trummer, "Metabilder zur Malerei«, in: Formalismus. Roland Goeschl, Heimo Zobernig, Lois Renner (Ausst. Kat. Wien 1997/98), Wien 1998, S. 131-143.

Unschärferelation. Fotografie als Dimension der Malerei (Ausst. Kat. Freiburg, Heidenheim, Saarbrücken 2000), hg. v. Stephan Berg, Ostfildern/Ruit 2000.

Benedetto Varchi, Vincenzio Borghini, Pittura e scultura nel Cinquecento, hg. v. Paola Barocchi, Livorno 1998.

Hans Walter, Satyrs Traum. Ein Gang durch die griechische Satyrlandschaft, München 1993.

Ways of Worldmaking (Ausst. Kat. Budapest 2001), hg. v. Harm Lux, Budapest 2001.

Paul Zanker, Eine Kunst für die Sinne. Zur Bilderwelt des Dionysos und der Aphrodite, Berlin 1998.

Andreas Zeising, »Imaginäre Wirklichkeiten. Fiktionale Orte in der neueren Fotografie«, in: heute bis jetzt, Ausst. Kat. Düsseldorf (2002), S. 22-33. 